Article

\title{
Identification, Characterization, and Mutational Analysis of a Probable KEAP1 Ortholog in Rice (Oryza sativa L.)
}

\author{
Yan-Hua Liu ${ }^{1}$, Meng Jiang ${ }^{1}{ }^{\oplus}$, Rui-Qing Li ${ }^{2}$, Rasbin Basnet ${ }^{1}$, Jian-Zhong Huang ${ }^{1,3}$, \\ Shi-Yong Song ${ }^{1}$ and Qing-Yao Shu ${ }^{1, *(D)}$ \\ 1 National Key Laboratory of Rice Biology and Zhejiang Key Laboratory of Crop Germplasm Resources, \\ Institute of Crop Sciences, Zhejiang University, Hangzhou 310058, China; 11616004@zju.edu.cn (Y.-H.L.); \\ mengjiang@zju.edu.cn (M.J.); 11416095@zju.edu.cn (R.B.); jzhuang@zju.edu.cn (J.-Z.H.); \\ shiyongsong@zju.edu.cn (S.-Y.S.) \\ 2 College of Agronomy, Anhui Agricultural University, Hefei 230036, China; 2018065@ahau.edu.cn \\ 3 Key Laboratory for Nuclear Agricultural Sciences of Zhejiang Province and Ministry of Agriculture and \\ Rural Affairs, Institute of Nuclear Agricultural Sciences, Zhejiang University, Zijingang Campus, \\ Hangzhou 310058, China \\ * Correspondence: qyshu@zju.edu.cn
}

Received: 27 July 2020; Accepted: 23 October 2020; Published: 27 October 2020

\begin{abstract}
The Kelch-like ECH-associated protein 1 (KEAP1)-nuclear factor E2-related factor 2 (NRF2) module is a key component in the detoxification and antioxidant system in animals, which plays crucial roles in cell homeostasis and cytoprotection, and consequently in carcinogenesis and disease development. However, this system seems to have diverged throughout evolution across different organisms, and the question of whether a similar system exists in plants has thus far remained unresolved. In this study, a KEAP1 ortholog was identified in rice (Oryza sativa L., OsKEAP1) and its properties were characterized via in silico and laboratory studies. To reveal OsKEAP1's function, two knockdown mutants, oskeap1-1 and oskeap1-2, were generated by targeted mutagenesis in the $5^{\prime}$ untranslated region (UTR) using the CRISPR-Cas9 system. In silico analysis showed that OsKEAP1 has a Kelch-repeat domain which is identical to those of animals and a plant-specific development and cell death (DCD) domain in place of the broad-complex, tramtrack, bric-a-brac (BTB) domain found in animals. Orthologs of OsKEAP1 are present across plant species and all have the DCD domain and the Kelch-repeat domain. OsKEAP1 was proven to be localized to both the cytoplasm and nucleus, in contrast to the exclusive cytoplasm localization of animal KEAP1. Single nucleotide insertions in the 5' UTR significantly reduced the transcription level of OsKEAP1 in the oskeap1-1 and oskeap1-2 mutants. The oskeap1 mutations greatly impaired plant growth and development, resulting in significant declines in a majority of agronomic and yield-related traits, i.e., plant height, panicle length, grain number per plant, and seed-set rate. The downregulation of OsKEAP1 increased the levels of $\mathrm{H}_{2} \mathrm{O}_{2}$, malondialdehyde, and proline while significantly decreasing the expression of two catalase genes in seedlings grown under normal and salt-stressed conditions. The changes in the above phenotypes are either positively or negatively correlated with the degree of OsKEAP1 downregulation. Altogether, we identified a probable KEAP1 ortholog in rice, revealed its unique subcellular localization, and demonstrated its important functions in vegetative and reproductive growth via regulation of the antioxidant response in plants.
\end{abstract}

Keywords: redox; seed germination; abscisic acid (ABA); OsKEAP1; Os01g0162500; Os05g0164900; Oryza sativa L.; CRISPR 


\section{Introduction}

Cells have developed a myriad of antioxidant mechanisms conferring defense against various redox stresses. The KEAP1-NRF2 module has been recognized as one of the prominent components in defense systems involving antioxidant response in animals [1-4]. KEAP1 (Kelch-like ECH-associated protein 1) is a negative regulator of NRF2 (NF-E2 related factor) and is responsible for ubiquitination regulation of NRF2 in response to oxidative and electrophilic stress [5,6]. NRF2 is constantly ubiquitinated by the complex between KEAP1 and Cullin3-based ubiquitin E3 ligase, thereby inhibiting NRF2 activity in normal conditions [6]. NRF2 has been identified as a potent transcription regulator of many cytoprotective genes that defend cells, tissues, and organisms against various xenobiotics and oxidative stress, including inflammatory diseases of the lung, liver, and kidney, as well as neurodegenerative diseases such as Alzheimer's and Parkinson's diseases [7-9].

The KEAP1-NRF2 pathway has also been found in other organisms from arthropods to mammals, although the evidence as to whether a similar KEAP1-NRF2 system exists in plants and other organisms remains either contradictory or elusive. Through bioinformatics-based evolutional analyses, Gacesa R. et al. [10] concluded that the KEAP1-NRF2 system exists in organisms from arthropods to mammals, but not in early Eukarya, fungi, Nematoda, and basal metazoa. However, the same group did identify fungal KEAP1 and NRF2 orthologs, although they were distant and quite diverged from animal ones [11]. This discrepancy could be explained by the different parameters and methods used for the homolog search. However, Gacesa R et al. [11] did not identify any KEAP1 and NRF2 orthologs in plants.

Plants are sessile- their development, growth, and survival are continuously challenged by internal and external stresses. Plants have evolved several mechanisms that enable them to use their metabolism by integrating primary metabolic products into vital processes. Reactive oxygen species (ROS) are one such example of metabolic products that play an important role in the regulation of plant growth and development [12]. It is known that ROS levels are determined by a tightly controlled balance between production and breakdown that is achieved via fine-tuning of complex antioxidant systems [13]. Because no close KEAP1 and NRF2 orthologs have been identified in plants via a classical homolog search [11], there have been no experimental studies on candidate genes in plants. Consequently, the KEAP1-NRF2 system, though widely existing in animals and other organisms for redox regulation, has never even been discussed in plants. A brief search for KEAP1 orthologs in the rice genome, however, revealed that there are hypothetical putative proteins with the Kelch-repeat domain. This initial finding inspired the authors to explore the potential KEAP1-NRF2 module in plants using rice as a model.

In the present study, we first identified a rice KEAP1 ortholog (OsKEAP1) and characterized its properties, such as conserved domains and subcellular localization. We then developed two downregulated OsKEAP1 mutants by means of targeted mutagenesis in its untranslated regions (UTRs) and investigated their agronomic and yield-related traits. Furthermore, we assessed the redox status and expression of a few genes known to be involved in redox under normal and abiotic-stressed conditions. Our results revealed that the downregulation of OsKEAP1 increased the level of $\mathrm{H}_{2} \mathrm{O}_{2}$, malondialdehyde, and proline and altered the expression of genes involved in the antioxidant response. Our present study thus reveals the function of OsKEAP1 in plant growth, development, and response to abiotic stresses and lays the basis for further investigation on whether there is a KEAP1-NRF2 module involved in ROS regulation in plants.

\section{Results}

\subsection{Identification and Characterization of the KEAP1 Ortholog in Rice}

Two KEAP1-like genes were identified in the rice genome (c.v. Nipponbare) with relatively high coverages: Os01g0162500 (54\%) and Os05g0164900 (46\%). In the NCBI database, the former is annotated to encode a hypothetical Kelch-type beta-propeller domain-containing protein, and the latter a Kelch 
repeat type 1 domain-containing protein, hence both have the Kelch domain, as found in KEAP1. However, the amino acid identity score of the rice proteins to KEAP1 was only $25.82 \%$ and $33.46 \%$, respectively (Figure 1A). Compared with KEAP1, both rice orthologs are much shorter in length but had more exons and introns. Both KEAP1 and its rice orthologs had relatively long untranslated regions (UTRs) at their $3^{\prime}$ termini (Figure 1B). The rice KEAP1 orthologs had five Kelch repeats, whereas KEAP1 had six Kelch repeats. At the $\mathrm{N}$ terminus, KEAP1 had a broad-complex, tramtrack, bric-a-brac (BTB-BACK) domain, whereas the rice orthologs had a development and programmed cell death (DCD) domain (Figure 1C). These characteristics suggest that KEAP1 and its rice orthologs might exhibit a similar basic function but respond to different development and environmental stimuli.

A

\begin{tabular}{|c|c|c|c|c|c|c|c|}
\hline \multirow{2}{*}{$\begin{array}{l}\text { Gene } \\
K E A P 1\end{array}$} & \multicolumn{2}{|c|}{ Gene ID } & \multirow{2}{*}{$\begin{array}{c}\text { Chromosome Location } \\
19: 10,486,125-10,503,558\end{array}$} & \multirow{2}{*}{$\begin{array}{c}\begin{array}{c}\text { Protein Length } \\
\text { (amino acid, aa) }\end{array} \\
624\end{array}$} & \multirow{2}{*}{$\begin{array}{c}\mathrm{MW}(\mathrm{Da}) \\
69,666\end{array}$} & \multirow{2}{*}{$\begin{array}{l}\text { Amino Acid } \\
\text { Identity (\%) } \\
-\end{array}$} & \multirow[t]{2}{*}{$E$ value } \\
\hline & $\begin{array}{l}\text { Gene Card: } \\
\text { GC19.M010486 }\end{array}$ & $\begin{array}{l}\text { MIMNo. } \\
606016\end{array}$ & & & & & \\
\hline OSKEAPI & $\begin{array}{c}\text { RAP: } \\
\text { Os01g0165200 }\end{array}$ & $\begin{array}{c}\text { MSU: } \\
\text { LOC_Os0lg07140 }\end{array}$ & 1: $3,362,001-3,367,658$ & 700 & 77,654 & 25.82 & $4 e-28$ \\
\hline umaaned & $\begin{array}{c}\text { RAP: } \\
\text { Os05g0164900 }\end{array}$ & $\begin{array}{c}\text { MSU: } \\
\text { LOC_OS05g07220 }\end{array}$ & $5: 3,813,306-3,819,768$ & 1123 & 122,975 & 33.46 & $4 e-42$ \\
\hline
\end{tabular}

B
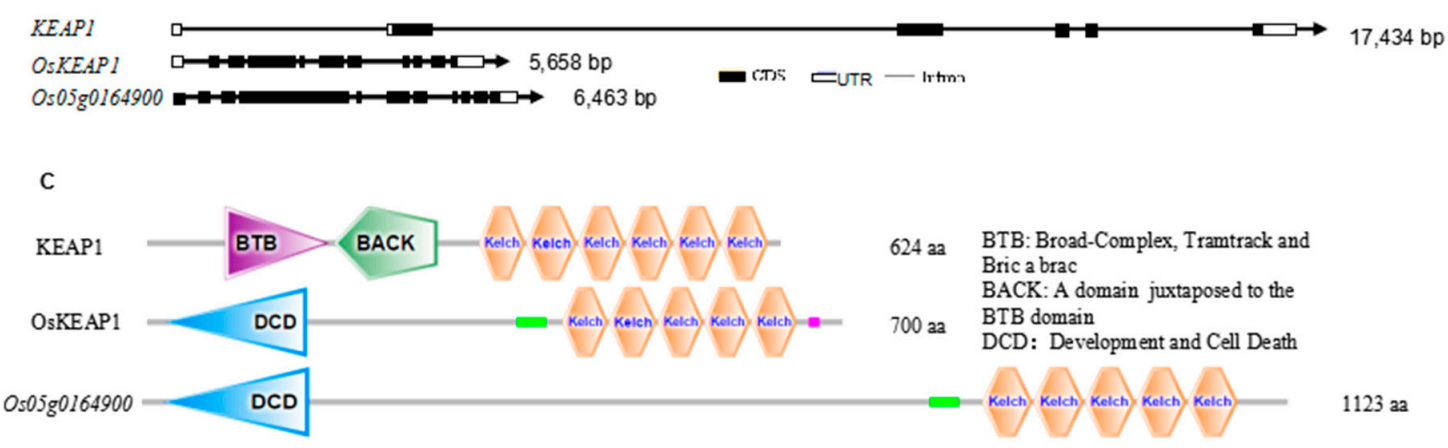

Figure 1. Gene information (A), structure (B), and protein features (C) of human KEAP1 and its orthologs in rice. Prediction of domains was performed at https://smart.embl.de/. The green and pink/red boxes represent the coiled-coil region and low complex region, respectively.

Because Os01g0162500 encodes a protein of 700 amino acids (aa), similar to KEAP1 (624 aa), while Os05g0164900 encodes a protein of 1123 aa, the peptide fragment between the DCD and Kelch domain of Os01g0162500 is far shorter than that of Os05g0164900. Hence, Os01g0162500 resembles KEAP1 more closely than Os05g0164900 does (Figure 1C). Therefore, Os01g0162500 was closer to KEAP1 than Os05g0164900 and we, therefore, deemed it to be the most probable KEAP1 ortholog in rice. Hereafter it is designated as OsKEAP1 and studied further.

In silico analysis showed that OsKEAP1 is mainly expressed in the inflorescence, anther, pistil, ovary, and embryo tissues (Figure S1). A search for proteins/peptides homologous to OsKEAP1/Os05g0164900 revealed 110 non-repeated sequences in plant species (Figure S2). Further analysis of a selection of KEAP1 orthologs in 10 crop plants showed that they are highly conserved in terms of protein length and domains, all having a DCD domain and 5-6 Kelch repeats (Figure S3).

To detect the subcellular localization of OsKEAP1, protoplasts transiently transformed with the OsKEAP1::GFP vector were observed under a fluorescence microscope. The results showed that the GFP signal from the control vector (pCAMBIA 1301) was found to be distributed throughout the cell, whereas the green fluorescent signal from GFP-fused proteins was transiently expressed in the nucleus and cytoplasm. The red fluorescence of NLS-mCherry overlapped with the green fluorescence, confirming the localization of OsKEAP1 in the nucleus, along with the cytoplasm (Figure 2). 


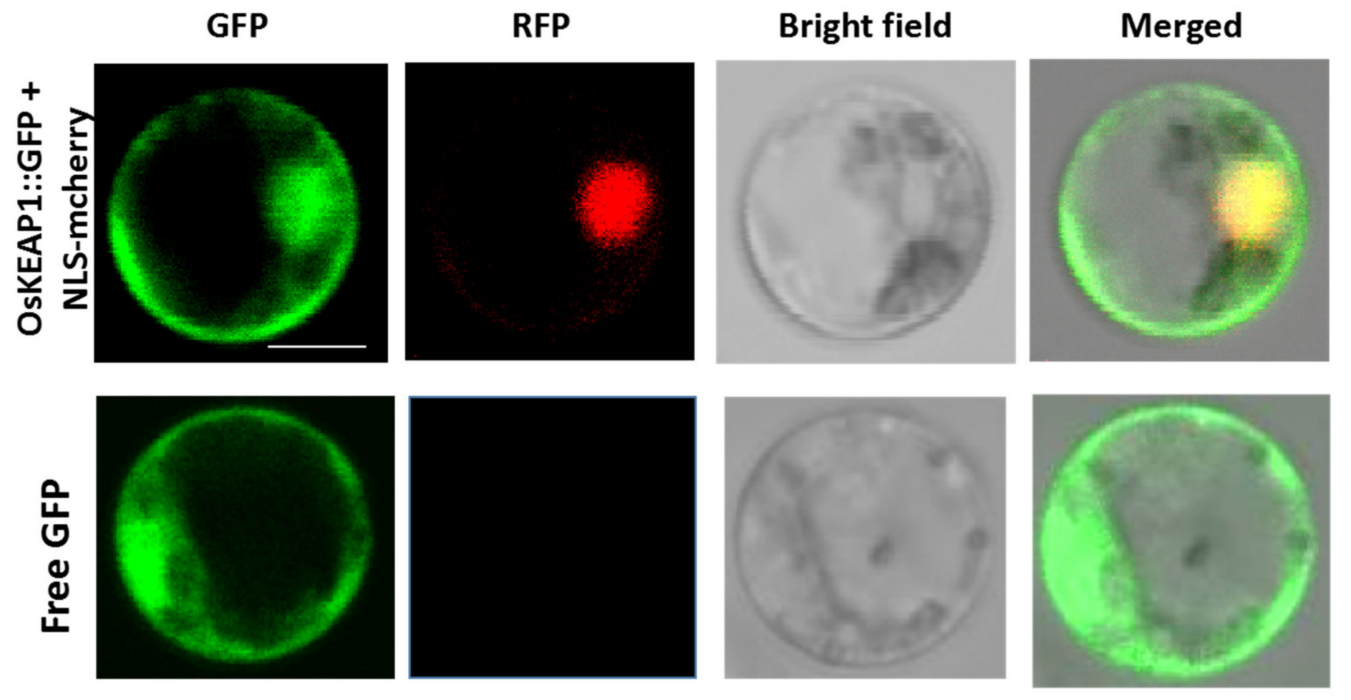

Figure 2. Transient expression of 35S:OsKEAP1:GFP fusion in rice protoplast. Protoplasts transfected with control vector (35S:GFP) have a bright GFP signal distributed throughout the cell, whereas those with 35S:OsKEAP1:GFP have a fluorescent signal (in green) localized in the nucleus and cytoplasm, confirmed by nuclear localization sequence (NLS) signal in the nucleus (in red). Bar $=5 \mu \mathrm{m}$.

\subsection{Targeted Mutagenesis of 5' UTR Downregulated OsKEAP1 Expression}

In our early trials, we failed to generate knockout oskeap 1 mutants by targeting its first exon. The Biogle Gene Research team, who produced a large mutant library by CRISPR/Cas9-based targeted mutagenesis of virtually all rice genes [14], also failed to identify mutants when targeting two sites in the 4th exon and one site in the 9th exon. Therefore, we opted to produce knockdown mutants by targeting the UTR (Figure $3 \mathrm{~A}$ ). Among the $\mathrm{T}_{0}$ plants, two homozygous 1-bp insertion mutants were identified, one with an A (oskeap1-1) and the other with a T (oskeap1-2) in OsKEAP1 (Figure 3B). The two plants were later developed into two transgene-free, stable mutant lines, which were named oskeap1-1 and oskeap1-2.

A

OsKEAP1 (Os01g0165200)

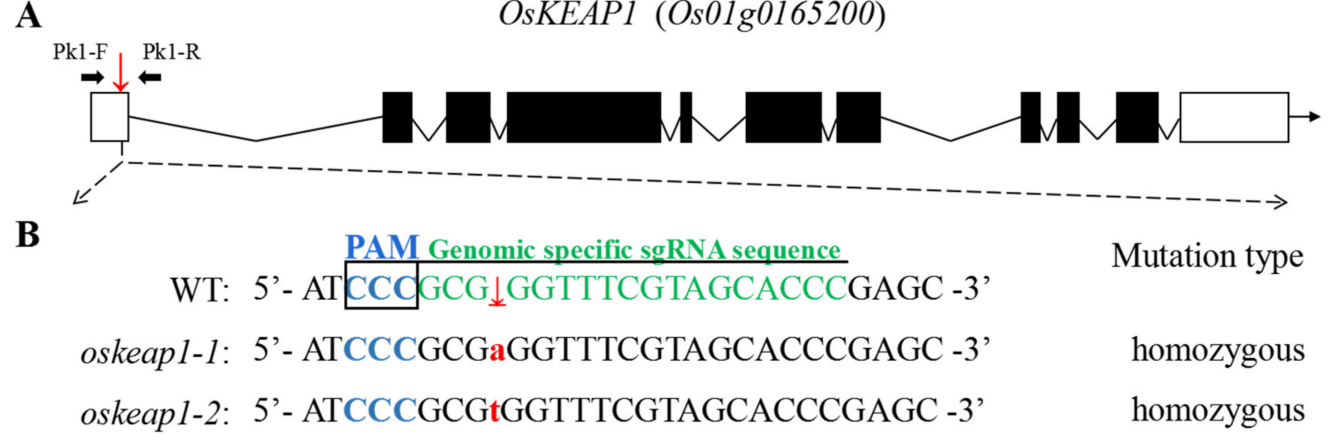

Figure 3. Targeted mutagenesis of OsKEAP1 using the CRISPR/Cas9 system. (A) Gene structure of OsKEAP1 with the sgRNA target shown by a frame; primers used for amplification of the fragment encompassing the target region (Pk1-F/R) are shown. (B) Sequence information around the target for the wild-type cultivar Xidao \#1 (wild-type (WT)) and its two oskeap1 mutants, the target sequence, and its PAM are highlighted.

To assess whether and how the two insertion mutations affected the expression of OsKEAP1, qRT-PCR was performed to detect the abundance of OsKEAP1 transcripts in the mutants and their wild-type parent Xidao \#1. The results showed that, at both the seedling and flowering stage, the two mutants had significantly less abundant OsKEAP1 transcripts than Xidao \#1 (Figure 4). Comparatively, the mutational effect of oskeap1-1 was more profound than that of oskeap1-2, with the abundance of 
OsKEAP1 transcripts being only $41.09 \%$ (oskeap1-1) and 77.39\% (oskeap1-2) that of Xidao \#1 at the seedling stage (Figure $4 \mathrm{~A}$ ). The mutational effect became more significant at the flowering stage, with the OsKEAP1 transcript level being only $29.77 \%$ and $50.46 \%$ that of the wild-type parent Xidao \#1, respectively (Figure $4 \mathrm{~B})$.
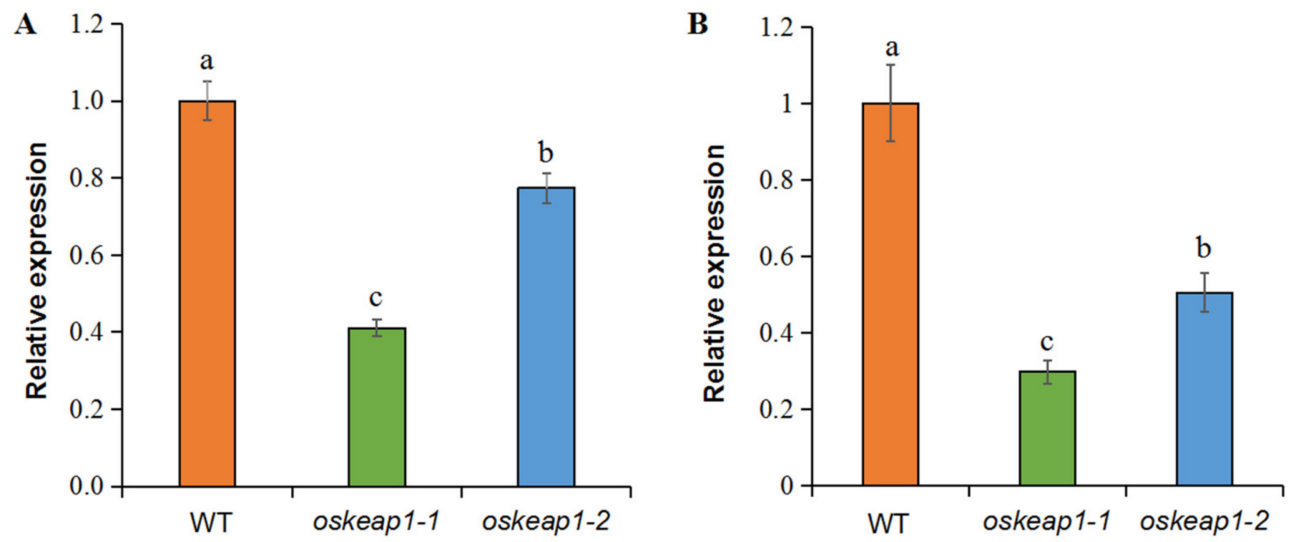

Figure 4. Relative expression of OsKEAP1 in leaf tissues at seedling (A) and heading stage (B) of rice cultivar Xidao \#1 (WT) and its two oskeap1 mutants. The expression levels of the two mutants are compared with their wild-type at the same stage. OsACTIN was used as an internal reference. Data are shown in mean \pm standard error of 3 biological repeats. Different letters $(a, b, c)$ represent significant difference at the 0.05 level.

\subsection{Impact of OsKEAP1 Down-Regulation on Plant Growth and Development}

The down-regulation of OsKEAP1 had a significant negative effect on plant growth right from seed germination to seed maturing. The seven-day-old seedlings of both oskeap1-1 and oskeap1-2 were already obviously shorter than those of the wild-type (WT). At the maturity stage, compared with the WT, the two mutants had significantly shorter plant height than the WT, with a decrease of $11.8 \%$ and $10.0 \%$, respectively (Figure 5A). Although the number of panicles per plant was not significantly different from that of the wild-type Xidao \#1 (Figure 5B), the panicle length of the two mutants was significantly reduced, by $22.9 \%$ and $7.7 \%$, respectively (Figure 5 C).

The oskeap 1 mutations had profound effects on plant reproduction. The total grain number per plant was significantly reduced in oskeap $1-1$ and oskeap $1-2$, by $27.5 \%$ and $14.9 \%$, respectively (Figure 5D). More dramatic reductions were observed in the seed-set rate, with reductions of $52.0 \%$ and 31.8\%, respectively, for oskeap1-1 and oskeap1-2 (Figure 5E). Consequently, the total seed yield per plot was significantly reduced in oskeap1-1 and oskeap $1-2$, by $62.2 \%$ and $39.3 \%$, respectively (Figure 5F). To examine whether the reduced seed-set rate was due to reduced male fertility, pollens were examined under a microscope after $\mathrm{KI}_{2} \mathrm{I}_{2}$ staining. The results showed that there were more unstained pollen grains in the two mutants than in wild-type parent Xidao \#1 (Figure 6), i.e., the rate of stained pollen grains was $84.46 \%, 87.74 \%$, and $96.14 \%$ in oskeap $1-1$, oskeap $1-2$, and wild-type Xidao \#1, respectively.

\subsection{Impact of OsKEAP1 Downregulation on Seed Germination and Sensitivity to Abscisic Acid (ABA)}

The oskeap1 mutations also seemed to have an adverse effect on seed development, resulting in grains with black spots and wrinkles (Figure 7A). When tested using an intact rice grain for germination, oskeap1-1 and oskeap1-2 had a significantly lower seed germination rate, at $43.06 \%$ and $54.17 \%$ that of the wild-type parent Xidao \#1 on day 6 (Figure 7B). To test whether the reduction of germination was related to seeds with black spots and wrinkles, rice grains were dehulled and brown rice grains without spots and wrinkles (normal looking grains) were selected for a germination test. No significant differences were observed between Xidao \#1 and its two oskeap1 mutants when grown on $\frac{1}{2}$ MS 
medium [15] (Figure 7C), suggesting the grains with black spots/wrinkles contributed to the reduction of seed germination.
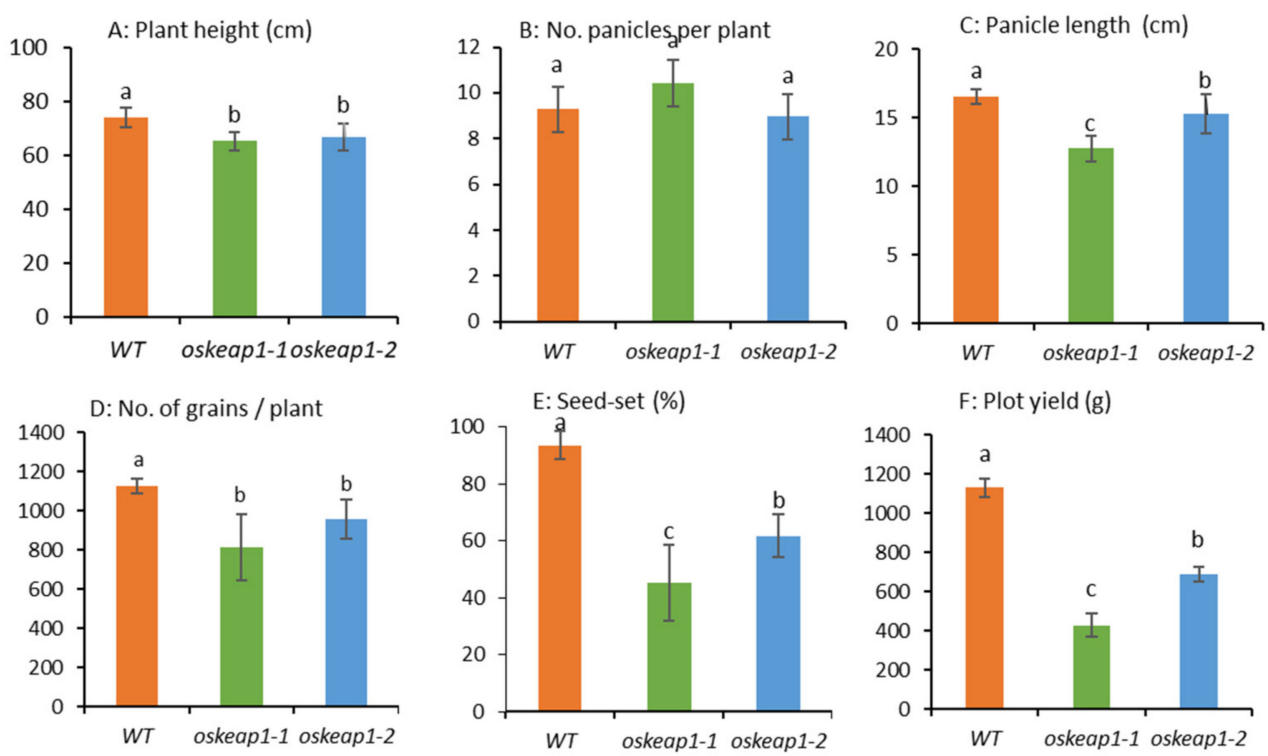

Figure 5. The agronomic and yield-related traits of rice wild-type cultivar Xidao \#1 (WT) and its two oskeap 1 mutants. Values are mean \pm SD of 3 replicates. Different letters represent significance at the 0.05 level.

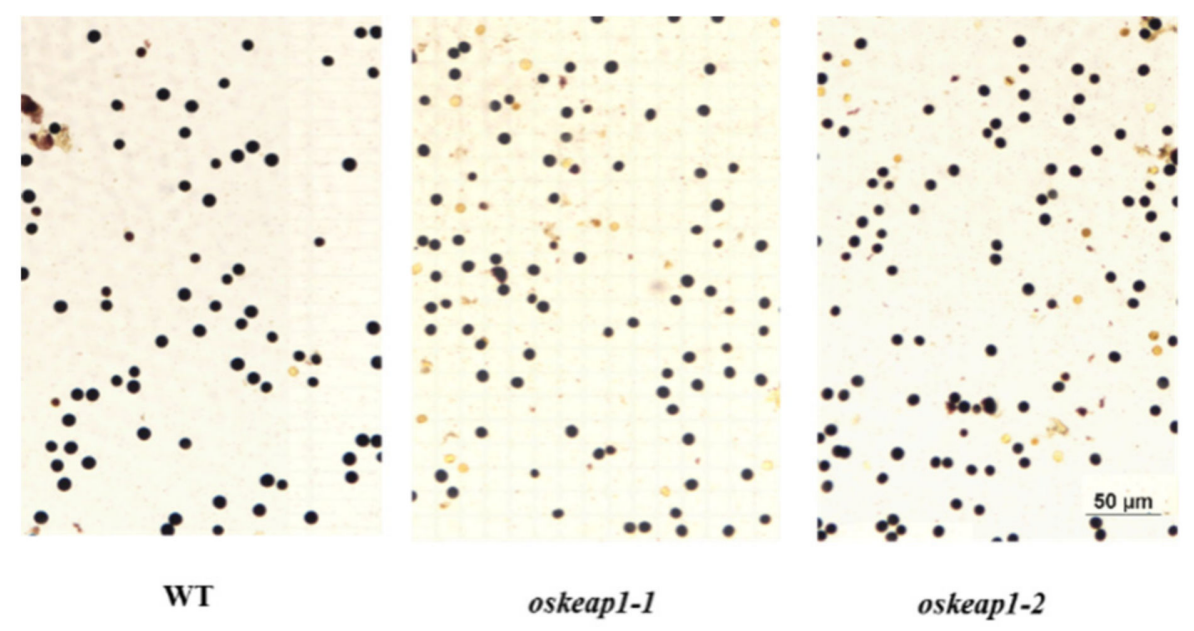

Figure 6. $\mathrm{K}-\mathrm{I}_{2}$-stained rice pollen grains of Xidao \#1 (WT) and its two oskeap1 mutants.

To further test whether OsKEAP1 is involved in the ABA signaling pathway, normal-looking mutant brown rice grains were tested for seed germination on $\frac{1}{2}$ MS medium with $1 \mu \mathrm{M}$ ABA. In this circumstance, oskeap 1 mutant seeds showed significantly lower germination rates, which suggests that they had become more sensitive to ABA than the wild-type Xidao \#1 (Figure 7D).

\subsection{Impact of OsKEAP1 Downregulation on Redox Regulation}

To test the influence of OsKEAP1 downregulation on redox status in rice, the $\mathrm{H}_{2} \mathrm{O}_{2}$ level was first measured in above-ground tissues of rice seedlings grown under normal and $\mathrm{NaCl}$-stressed conditions. Under both normal and salt stress conditions, the two mutants had significantly higher $\mathrm{H}_{2} \mathrm{O}_{2}$ levels than the WT (Figure 8A). Salt treatment significantly increased the $\mathrm{H}_{2} \mathrm{O}_{2}$ level in both the WT and its two oskeap1 mutants (Figure 8A). 

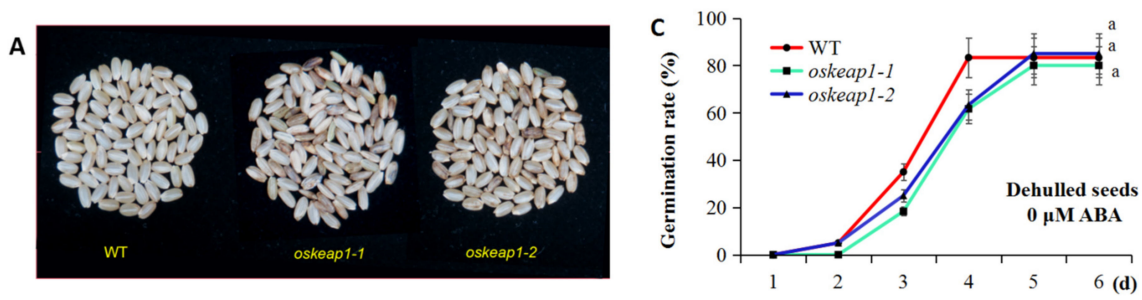

B
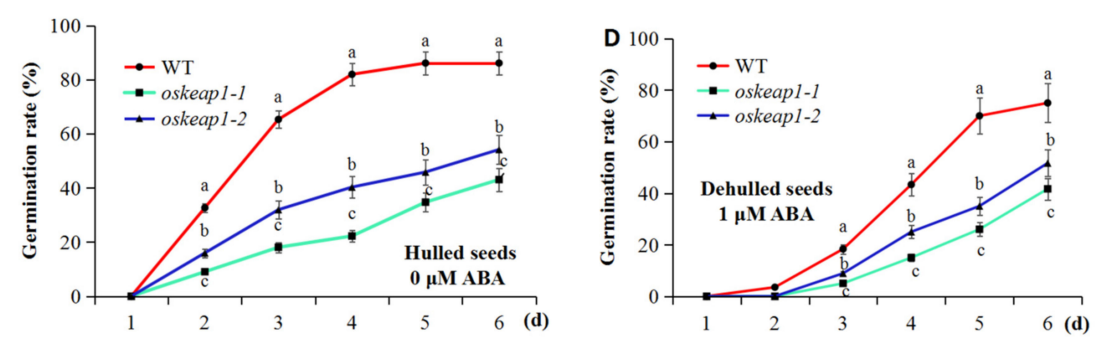

Figure 7. The phenotype of dehulled rice grains of wild-type Xidao\#1 (WT) and its oskeap1 mutants (A) and germination rate of hulled seeds (B) and dehulled seeds (brown rice) on medium without (C) or with (D) $1 \mu \mathrm{M}$ abscisic acid (ABA). Data from the same observation date with different letters $(\mathrm{a}, \mathrm{b}, \mathrm{c})$ are significantly different at the 0.05 level.
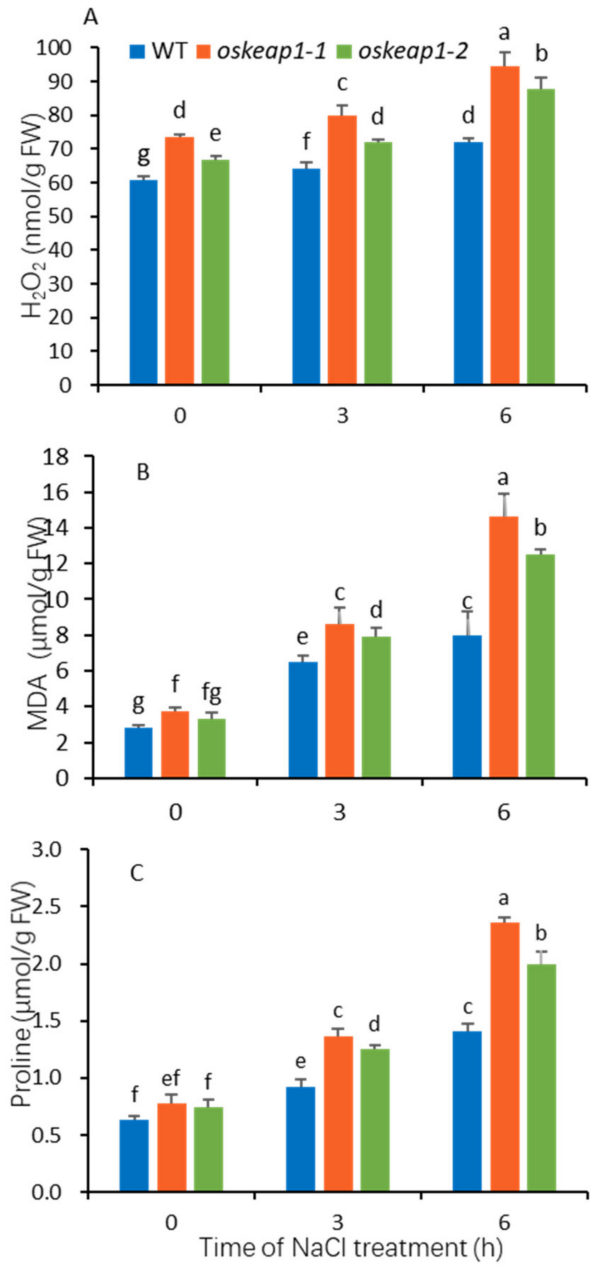

Figure 8. The content of $\mathrm{H}_{2} \mathrm{O}_{2}$, malondialdehyde (MDA), and proline in the above-ground tissues of Xidao \#1 (WT) and its oskeap1 mutants. Ten-day-old seedlings were treated with $200 \mathrm{mM} \mathrm{NaCl}$ for 3 and $6 \mathrm{~h}$. Data with different letters (a,b,c, etc.) are significantly different at the 0.05 level. 
Malondialdehyde (MDA) and proline are two indicators of redox status and plants often have increased levels of these two chemicals after being subjected to stress treatment. Under normal conditions, the two oskeap1 mutants had MDA and proline levels similar to that of the WT, except that the MDA content of oskeap1-1 was significantly different from that of the WT (Figure 8B). Salt treatment significantly increased the levels of MDA and proline in both the WT and its two oskeap1 mutants, with the same trend as $\mathrm{H}_{2} \mathrm{O}_{2}$ (Figure $8 \mathrm{~B}, \mathrm{C}$ ).

To understand the biological mechanism leading to the redox changes in the oskeap 1 mutants, the expression of OsKEAP1, Os05g0164900, and two catalase genes was investigated in leaf tissue of plants grown under normal conditions and subjected to salt $(\mathrm{NaCl}, 200 \mathrm{mM})$ and $\mathrm{H}_{2} \mathrm{O}_{2}(10 \mathrm{mM})$ treatment (Figure 9).
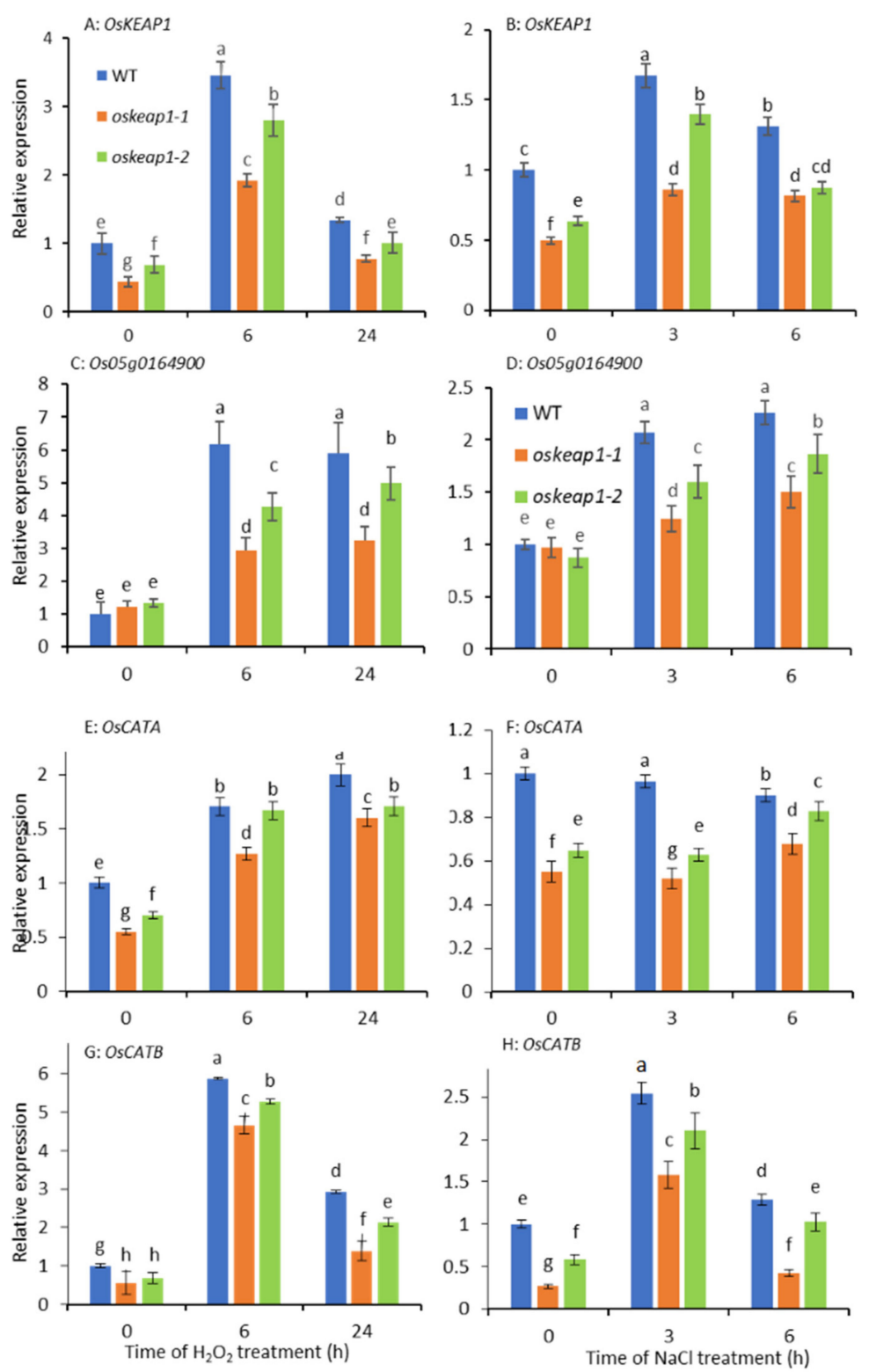

Figure 9. Relative expression of OsKEAP1, Os05g0164900, and two catalase genes (OsCATA and OsCATB) in leaf tissues of rice cultivar Xidao \#1 (WT) and its two oskeap1 mutants grown under normal conditions and subjected to salt $(\mathrm{NaCl}, 200 \mathrm{mM})$ and $\mathrm{H}_{2} \mathrm{O}_{2}(10 \mathrm{mM})$ treatment. OsACTIN was used as an internal reference and the expression levels were all compared with that of the WT grown under normal conditions (set as 1). Data are shown as mean \pm standard error of 3 biological repeats. Data with different letters $(\mathrm{a}, \mathrm{b}, \mathrm{c}, \mathrm{etc}$.$) represent significance at the 0.05$ level. 
The expression of OsKEAP1 seemed to be responsive to both $\mathrm{H}_{2} \mathrm{O}_{2}$ and salt treatment, and the mRNA transcript levels of the two oskeap1 mutants were significantly lower than those of the WT grown either under normal conditions or in media supplemented with $\mathrm{H}_{2} \mathrm{O}_{2}$ (Figure 9A) and salt (Figure 9B). A similar trend was observed for Os05g0164900, i.e., its expression was also responsive to these two stresses (Figure 9C,D). The OsKEAP1 mutations did not affect the expression of Os05g0164900 under normal conditions, but significantly lowered its expression under both stresses (Figure 9C,D).

To examine whether the oskeap1 mutations affected the expression of genes involved in ROS degradation, the expression of two catalase genes, OsCATA and OsCATB, was investigated. The two genes seemed to have different responses to salt and $\mathrm{H}_{2} \mathrm{O}_{2}$ treatment. The former is responsive only to $\mathrm{H}_{2} \mathrm{O}_{2}$ treatment (Figure 9E,F), whereas the latter is responsive to both treatments (Figure 9G,H). The two oskeap1 mutations seemed to have significantly repressed the expression of OsCATA and OsCATB, irrespective of treatment (Figure 9E-H).

\section{Discussion}

As a starting point to explore whether there is a ROS regulatory pathway in plants similar to the KEAP1-NRF2 pathway found in animals and other organisms, the present study first identified the most likely KEAP1 ortholog in rice (OsKEAP1), and then generated two knockdown mutants by targeted mutagenesis on its $5^{\prime}$ UTR. Further examination revealed that downregulation of OsKEAP1 impaired plant growth and development, altered stress response and ROS accumulation in seedlings, and increased sensitivity to ABA in seed germination. This study not only identified and characterized the first probable KEAP1 ortholog in plants but also served as an example of a unique way of generating knockdown mutants by means of UTR editing.

The failure to generate knockout oskeap 1 mutants by us and other groups suggests that OsKEAP1 may play an indispensable role in rice growth and development and its complete knockout might be lethal. Based on this reasoning, we generated two downregulated mutants by targeting its $5^{\prime}$ UTR, which is known to regulate mRNA stability and translation [16]. Both oskeap1 mutants had a 1-bp insertion in the same position of the $5^{\prime}$ UTR (Figure 3B). The former had a significantly greater negative effect than the latter (Figure 4). This seems to be consistent with their theoretical effect on the secondary structure of mRNA (Figure S4) - the A insertion in oskeap1-1 is expected to result in the disappearance of the downstream loop, whereas the T insertion in oskeap1-2 would only lead to an enlarged downstream loop. The different downregulation level of OsKEAP1 in the two oskeap1 mutants indeed enabled us to quantitatively link the function of OsKEAP1 to multiple phenotypes, as evidenced by the changes in almost all phenotypes, from agronomic traits and seed germination to responses to $\mathrm{H}_{2} \mathrm{O}_{2}$ and salt treatments, in proportion to the downregulation level in the two mutants.

OsKEAP1 was initially identified through in silico analysis in the present study. Because its amino acid identity to KEAP1 is relatively low (Figure 1A) and previous studies also did not identify a close KEAP1 ortholog in plants, the evidence of OsKEAP1 as the ortholog of KEAP1 in rice may not be sufficient. However, our study did prove that OsKEAP1 is involved in redox regulation, based on the following observations. First, OsKEAP1 has the key domain of KEAP1—the Kelch repeats domain (Figure 1C) - and the expression of OsKEAP1 is responsive to $\mathrm{H}_{2} \mathrm{O}_{2}$ and salt treatment. Second, we demonstrated that the two mutants had significantly greater levels of $\mathrm{H}_{2} \mathrm{O}_{2}, \mathrm{MDA}$, and proline than their wild-type Xidao \#1 parent (Figure 8), suggesting that the downregulation of OSKEAP1 impaired the ability to clean ROS in seedlings. Third, the expression of OsCATA and OsCATB was significantly downregulated in the two oskeap 1 mutants grown under normal conditions and under stresses (Figure 9C-F), suggesting that the ROS increase in the two mutants is related to the altered regulation of ROS response. However, the working mechanism of KEAP1-NRF2, if it exists in rice, seems already to be different from that found in animals. In humans and animals, the downregulation of KEAP1 would increase the expression of its partner, NRF2, and consequently activate the expression of downstream antioxidant genes [17]. In the present study, we observed the opposite trend-the $\mathrm{H}_{2} \mathrm{O}_{2}$ level was increased in the two mutants (Figure 8A), whereas the expression of two catalase genes was 
significantly decreased (Figure 9E-H). Further studies are needed to reveal the mechanism and explain how OsKEAP1 works differently from KEAP1 in ROS regulation. For this, the following observations may provide some clues: KEAP1 has a BTB-BACK domain, whereas OsKEAP1 has a DCD domain at the $\mathrm{N}$ terminus (Figure 1C), and OsKEAP1 is located in both the cytoplasm and nucleus (Figure 2), whereas animal KEAP1 is found only in the cytoplasm [18].

OsKEAP1 seems to have orthologs among different plant species, all of which have DCD domains and the Kelch repeats (Figures S2 and S3). DCD is a plant-specific domain involved in phytohormone response, plant development, and programmed cell death [19]. Indeed, a simple in silico search of NRF2 homologs in rice revealed OsABI5 [20], the rice ortholog of the Arabidopsis gene ABA Insensitive 5 [21]) to be the top candidate, though it also has a lower amino acid identity $(<30 \%)$ to NRF2. ABI5 is a basic leucine zipper transcription factor and plays a broad role in the regulation of seed germination and early seedling growth, as well as regulation of the response to abiotic stresses [22]. This finding is not only in line with the potential role of OsKEAP1 in phytohormone (abscisic acid, ABA) response, as shown by the DCD domain, it could also explain the increased sensitivity of the oskeap 1 mutants to ABA (Figure 7D) because downregulation of OsKEAP1 is expected to upregulate OsABI5.

OsKEAP1 is differently expressed in various plant organs and tissues (Figure S1), hence its mutational effect on different traits could be quite different. In addition to the anthers, OsKEAP1 also is highly expressed in the inflorescence, pistil, and ovary. This might be the reason underlying the great decrease of seed-set in the two mutants, which could not be explained merely by pollen fertility reduction (Figure 6). We speculate that the viability of megaspores and other female organs also may have been negatively affected by the downregulation of OsKEAP1 in the two mutants, although more experiments are needed to validate this.

Os05g0164900 is a close paralog of OsKEAP1. It has both DCD and Kelch-repeat domains (Figure $1 \mathrm{C}$ ) and its expression is responsive to salt and $\mathrm{H}_{2} \mathrm{O}_{2}$ treatment (Figure 9C,D). Moreover, when subjected to salt and $\mathrm{H}_{2} \mathrm{O}_{2}$ treatment, its expression level was significantly lower in the two mutants than their WT parents, in a similar trend to that of OsKEAP1 (Figure 9C,D). These features suggest that Os05g0164900 could be another potential KEAP1 ortholog in rice.

\section{Materials and Methods}

\subsection{In Silico Identification and Characterization of KEAP1 Orthologs in Plants}

The amino acid sequences of human KEAP1, downloaded from NCBI [23], were used as a query for a BLAST search in the NCBI database to search for the KEAP1 orthologs in rice. Multiple databases were further searched for the identification of KEAP1 orthologous genes in rice, such as Gramene [24] and RAPDB [25]. The amino acid sequences of KEAP1 orthologs of other plant species such as maize and sorghum were downloaded from Gramene [24] or OrthoDB [26]. The conserved domains of KEAP1 were analyzed using the Unipro and SMART databases. The secondary structure of the UTR of KEAP1 was predicted in UNAFold [27]. The tissue-specific expression profile of genes in rice was obtained from RiceXPro [28].

\subsection{Construction of CRISPR/Cas9 Vector}

The optimal base-pairing sequences of sgRNA targeting the 5' UTR of OsKEAP1 were identified using the CRISPR-P 2.0 tool (http://crispr.hzau.edu.cn/CRISPR2/). The following sgRNA target oligo was chosen and a BsaI recognition site was added on both ends of KEAP1-F and its complementary sequence KEAP1-R and synthesized from Tsingke (Hangzhou, China). The two oligos were annealed using Annealing Buffer 5X (Beyotime) and ligated to linearized pHUN4c12 (digested by BsaI, NEB). The recombinant vector (pHUN4c12-K5U) was transformed into Escherichia coli and the insertion of oligos was confirmed by sequencing (TSINGKE, Hangzhou, China). The pHUN4c12-K5U vector was then transformed into Agrobacterium (strain EHA105). 
Healthy seeds of the japonica rice variety Xidao \#1 were de-hulled and surface sterilized using $70 \%$ ethanol for $1 \mathrm{~min}$ and $1 \% \mathrm{NaClO}$ for 30-40 min, followed by rinsing with sterile distilled water $4-5$ times. These seeds were then cultivated on a $2 \mathrm{~N} 6$ callus-inducing medium [29] for 1 month at $28^{\circ} \mathrm{C}$ and a 16 -h photoperiod. When the diameter of the callus reached $\sim 4 \mathrm{~mm}$, the calli were transformed with pHUN4c12-K5U via Agrobacterium-mediated transformation. The transformed calli were then grown on selective media supplemented with hygromycin and tetracycline according to the method of Liu et al. [30]. Surviving calli were grown into plantlets in regeneration media, and later transplanted in soil and grown into $\mathrm{T}_{0}$ plants.

\subsection{Identification and Development of OsKEAP1 Mutants}

The genomic DNA of $\mathrm{T}_{0}$ plants was extracted by the modified CTAB (cetyltrimethylammonium bromide) method [30] and the targeted region of OsKEAP1 was PCR amplified using primer pair Pk1-F and Pk1-R, with the following program: $95{ }^{\circ} \mathrm{C}, 2 \mathrm{~min}, 35$ cycles of a three-step reaction including $95{ }^{\circ} \mathrm{C}$ for $30 \mathrm{~s}, 55^{\circ} \mathrm{C}$ for $30 \mathrm{~s}$ and $72{ }^{\circ} \mathrm{C}$ for $40 \mathrm{~s}$, and a final extension at $72{ }^{\circ} \mathrm{C}$ for $8 \mathrm{~min}$. The PCR products were examined using high resolution melting (HRM) analysis, according to the method of $\mathrm{Li}$ et al. [31], and putative mutant samples were then sequenced to detect mutations in the target region. Seeds from mutant $T_{0}$ plants were harvested and grown into $T_{1}$ plants and analyzed further for the confirmation of mutations in the UTR and absence of transfer DNA (T-DNA) according to the method of Basnet et al. [32].

\subsection{Real-Time Quantitative-PCR and Subcellular Localization}

For real-time quantitative PCR (qPCR) analysis, total RNA was extracted from above-ground tissues of 10-day-old rice seedlings using an RNAprep pure Plant Kit (Tiangen, Beijing, China) and reverse transcribed using a PrimerScript RT reagent kit with genomic DNA eraser (Takara, Dalian, China). A Hieff ${ }^{\mathrm{TM}}$ qPCR SYBR ${ }^{\circledR}$ Master Mix (Yeasen, Shanghai, China) was used for qPCR in a Roche Illuminator (Penzberg, Germany). The primer pair KPqpcr-F and KPqpcr-R was used for qPCR of OsKEAP1, qKP2-F, and qKP2-R for Os05g0164900, qCATA-F, and qCATA-R for OsCATA, and qCATB-F and qCATB-R for OsCATB (Table 1). The rice Actin gene was used as an internal reference. Three biological replicates were taken for each treatment and the relative expression levels were calculated using the $2^{-\triangle \triangle C T}$ analysis method.

Table 1. List of primers used in this study.

\begin{tabular}{|c|c|c|}
\hline Name of Primers & Sequences $\left(5^{\prime}-3^{\prime}\right)$ & Remarks \\
\hline KEAP1-F & ggcaGGGTGCTACGAAACCCGC & OsKEAP1 sgRNA synthesize \\
\hline $\begin{array}{l}\text { Pk1-F } \\
\text { Pk1-R }\end{array}$ & $\begin{array}{l}\text { GGTTGATCGATGCTTGCTGC } \\
\text { CACCAATCGCGACCAAATCG }\end{array}$ & $\begin{array}{l}\text { For oskeap1 mutant identification and high } \\
\text { resolution melting (HRM) analysis }\end{array}$ \\
\hline $\begin{array}{l}\text { KPqpcr-F } \\
\text { KPqpcr-R } \\
\text { qKp2-F } \\
\text { qKp2-R } \\
\text { qCATA-F } \\
\text { qCATA-R } \\
\text { qCATB-F } \\
\text { qCATB-R }\end{array}$ & $\begin{array}{c}\text { CAAGCACTGGCCAGCTTAAT } \\
\text { GATTAGCGCGAACAGGAGCA } \\
\text { GCAGCCCGTCTATGATGAACTCTC } \\
\text { AGAAACCCTCCCTTGGGTCATAC } \\
\text { TCCCAGTGTGATGAGTCGTTGG } \\
\text { TCTTCACATGCTTGGCTTCACG } \\
\text { TCCTACTGGTCGCAGTGTGATG } \\
\text { TTTCAGGTTGAGACGTGAAGCC }\end{array}$ & q-PCR analysis \\
\hline $\begin{array}{l}\text { KpGFP F } \\
\text { KpGFP R }\end{array}$ & $\begin{array}{l}\text { gaattcATGGGTGCTGGAAAGAAGACTCA } \\
\text { ggatccCAATGGCAACGGCGCATGC }\end{array}$ & Complementary DNA amplification \\
\hline $\begin{array}{l}\text { HYG-F } \\
\text { HYG-R }\end{array}$ & $\begin{array}{l}\text { AGAAGAAGATGTTGGCGACCT } \\
\text { GTCCTGCGGGTAAATAGCT }\end{array}$ & Hygromycin marker primer \\
\hline
\end{tabular}

The complementary DNA (cDNA) of OsKEAP1 was obtained by PCR amplification using the forward primer KpGFP-F containing the EcoRI recognition site, and the reverse primer KpGFP-R containing the BamHI recognition site. The PCR product was cloned into the linear vector PTZM28-GFP 
(digested with EcoRI and BamHI) to produce a fusion gene with a green fluorescence protein (GFP) under the control of the cauliflower mosaic virus (CaMV) 35s promoter (named the OsKEAP1::GFP vector).

Rice protoplasts were isolated from 10-day-old rice seedlings. The OsKEAP1::GFP was co-transformed with a mCherry-labeled nucleus marker (NLS-mCherry) into the rice protoplasts using polyethylene glycol [33] and incubated for 12-16 h. The pCAMBIA 1301 GFP vector under the control of the CaMV $35 \mathrm{~S}$ promoter was used as a control. The subcellular localization of fluorescent proteins in protoplasts was observed under an LSM780 fluorescence confocal microscope.

\subsection{Agronomic and Seed Germination Tests of OsKEAP1 Mutants}

Transgene-free homozygous mutant $T_{2}$ lines were developed from transgene-free $T_{1}$ plants and were tested in plots for their agronomic performance along with their wild-type parental line Xidao \#1, at the Zhejiang rice breeding station in Hangzhou. The two mutant lines and their parent line Xiadao \#1 were grown in a randomized design with three replicates. In each replicate, $48(6 \times 8)$ plants of each line were grown as a plot. Agronomic data, including tiller numbers, plant height, thousand-grain weight, seed setting rate, and other agronomic traits, were collected at the grain maturity stage [32]. Pollen fertility was studied by the iodine staining method [34] and observed under a microscope. Plot yield is the total grain yield of each replicate.

For regular germination tests, grains were soaked in water at $30^{\circ} \mathrm{C}$ for $48 \mathrm{~h}$ and then transferred onto wet filter paper for germination at room temperature. The test was performed in triplicate, each with 300 grains. The number of germinated seeds was recorded daily for 6 days. For assessment of the effect of $A B A$ on seed germination, grains were dehulled and normal-looking brown rice grains were selected for testing. The rice grains were surface sterilized by rinsing with $75 \%$ ethanol for $30-60 \mathrm{~s}$, followed by soaking in $1 \% \mathrm{NaClO}$ solution for $40 \mathrm{~min}$. After rinsing in water $4-5$ times, the sterilized grains were cultured on $1 / 2 \mathrm{MS}$ medium [15] supplemented with 0 and $1 \mu \mathrm{M}$ ABA. The test was performed in triplicate, each with 60 grains.

The sequence information for all PCR primers and DNA oligos is listed in Table 1.

\subsection{Seedling Growth}

For testing of the respective redox states and responses to stresses, healthy and uniform seeds of Xidao \#1 and its two oskeap 1 mutants were soaked in distilled water for $48 \mathrm{~h}$ at $30^{\circ} \mathrm{C}$ in an incubator and then germinated on wet filter paper in darkness for $48 \mathrm{~h}$. Then the germinating seeds were grown in $1 / 2 \mathrm{MS}$ liquid medium for 10 days in a growth chamber. The temperature was set at $24{ }^{\circ} \mathrm{C} / 16^{\circ} \mathrm{C}$ for day/night with $16 \mathrm{~h}$ of light, with an irradiance of $300 \mu \mathrm{mol} \mathrm{m}^{-2} \mathrm{~s}^{-1}$ and relative humidity of $60-70 \%$. Uniform 10-day-old seedlings were used for subsequent experiments. For salt stress treatment, $\mathrm{NaCl}$ was added into a liquid medium at a final concentration of $200 \mathrm{mM}$ and grown for up to $6 \mathrm{~h}$, and for oxidative treatment, $\mathrm{H}_{2} \mathrm{O}_{2}$ was added at the final level of $10 \mathrm{mM}$ and grown-up for $24 \mathrm{~h}$ before seedlings were taken for measurement.

\subsection{Measurement of $\mathrm{H}_{2} \mathrm{O}_{2}, \mathrm{MDA}$ and Proline Content}

The $\mathrm{H}_{2} \mathrm{O}_{2}$ level was determined in seedlings without roots using an $\mathrm{H}_{2} \mathrm{O}_{2}$ detection kit (Solarbio, Shanghai, China), according to the manufacturer's instructions, and the optical density was recorded at $415 \mathrm{~nm}$. Samples ( $\sim .1 \mathrm{~g})$ were prepared in liquid nitrogen and immediately used for the determination of $\mathrm{H}_{2} \mathrm{O}_{2}$ content. Six biological replicates were performed for each treatment.

The content of MDA was determined according to the method of Jiang et al. [35]. About $0.1 \mathrm{~g}$ tissues were mixed with $10 \%$ trichloroacetic acid and centrifuged at 10,000 $\mathrm{g}$ for $20 \mathrm{~min}$. The supernatant was blended with the same amount of thiobarbituric acid and incubated at $95^{\circ} \mathrm{C}$ for $40 \mathrm{~min}$. After being quickly cooled on ice, the mixture was centrifugated for $10 \mathrm{~min}$ at $12,000 \times \mathrm{g}$. The absorbances of the supernatant were determined at 450,532, and $600 \mathrm{~nm}$, respectively.

The content of proline was measured following the protocol published by He et al. [36]. About $0.1 \mathrm{~g}$ tissues were homogenized with $3 \%$ sulfosalicylic acid and centrifuged at 10,000 $\mathrm{g}$ for $20 \mathrm{~min}$. 
The supernatant was mixed with an equal volume of acidic ninhydrin and glacial acetic acid and incubated in boiling water for $50 \mathrm{~min}$. After being quickly cooled on ice, the homogenate was mixed with toluene, the aqueous phase was collected and their absorbance was measured at $520 \mathrm{~nm}$. The proline content was calculated using a standard curve.

\section{Conclusions}

In this study, we identified a probable KEAP1 ortholog in rice (OsKEAP1) based on its amino acid identity and domain features and demonstrated its involvement in ROS accumulation and response. We demonstrated that the downregulation of OsKEAP1 could significantly and negatively affect agronomic performance, including yield-related traits and increased sensitivity to ABA in seed germination. Based on elevated levels of $\mathrm{H}_{2} \mathrm{O}_{2}$, MDA, and proline and decreased expression of two catalase genes in the oskeap1 mutant seedlings, it was deduced that OsKEAP1 is involved in ROS regulation in rice, but in a way that is different from that of KEAP1. More studies are worthwhile to reveal the roles and working mechanisms of OsKEAP1 in rice growth, development, and responses to various internal and external stresses.

Supplementary Materials: The following are available online at http://www.mdpi.com/2223-7747/9/11/1450/s1, Figure S1: Relative expression of OsKEAP1 in different rice tissues, Figure S2: Molecular Phylogenetic analysis of KEAP1 orthologs in plants, Figure S3: Domain analysis of KEAP1 ortholog in 10 plant species, Figure S4: Prediction of secondary RNA structure of two OsKEAP1 mutants, each with a single nucleotide insertion in its untranslated regions.

Author Contributions: Q.-Y.S., S.-Y.S., R.-Q.L. and M.J. contributed to the overall research structure design and modification of the manuscript. Y.-H.L. planned the research work, doing laboratory work and data analysis. R.B. and J.-Z.H. contributed in manuscript modification. All authors have read and agreed to the published version of the manuscript.

Funding: This study was supported by a grant from the National Key Research and Development Program of China (2016YFD0102103); and in part by the International Atomic Energy Agency via a Coordinated Research Project (D23032, contract No. 23042).

Acknowledgments: Sincere thanks to researchers and staff from Zhijiang Seed Industry Co., LTD, Jiaxing Agricultural Research Institute, Wuxi Hupper Seed Industry Technology Research Institute Co., LTD, and Hainan south breeding base of Zhejiang University for their help in field cultivation of research materials.

Conflicts of Interest: The authors declare no conflict of interest.

\section{References}

1. Jain, A.; Lamark, T.; Sjøttem, E.; Larsen, K.B.; Awuh, J.A.; Øvervatn, A.; McMahon, M.; Hayes, J.D.; Johansen, T. p62/SQSTM1 is a target gene for transcription factor NRF2 and creates a positive feedback loop by inducing antioxidant response element-driven gene transcription. J. Biol. Chem. 2010, 285, 22576-22591. [CrossRef] [PubMed]

2. Fan, W.; Tang, Z.; Chen, D.; Moughon, D.; Ding, X.; Chen, S.; Zhu, M.; Zhong, Q. Keap1 facilitates p62-mediated ubiquitin aggregate clearance via autophagy. Autophagy. 2010, 6, 614-621. [CrossRef] [PubMed]

3. Lau, A.; Wang, X.-J.; Zhao, F.; Villeneuve, N.F.; Wu, T.; Jiang, T.; Sun, Z.; White, E.; Zhang, D.D. A Noncanonical mechanism of Nrf2 activation by autophagy deficiency: Direct interaction between Keap1 and p62. Mol. Cell. Biol. 2010, 30, 3275-3285. [CrossRef] [PubMed]

4. Komatsu, M.; Kurokawa, H.; Waguri, S.; Taguchi, K.; Kobayashi, A.; Ichimura, Y.; Sou, Y.S.; Ueno, I.; Sakamoto, A.; Tong, K.I.; et al. The selective autophagy substrate p62 activates the stress responsive transcription factor Nrf2 through inactivation of Keap1. Nat. Cell Biol. 2010, 12, 213-223. [CrossRef] [PubMed]

5. Leiser, S.F.; Miller, R.A. Nrf2 signaling, a mechanism for cellular stress resistance in long-lived mice. Mol. Cell. Biol. 2010, 30, 871-884. [CrossRef] [PubMed]

6. Uruno, A.; Motohashi, H. The Keap1-Nrf2 system as an in vivo sensor for electrophiles. Nitric Oxide Biol. Chem. 2011, 25, 153-160. [CrossRef]

7. Singh, A.; Misra, V.; Thimmulappa, R.; Lee, H.; Ames, S.; Hoque, M.; Herman, J.; Baylin, S.; Sidransky, D.; Gabrielson, E.; et al. Dysfunctional KEAP1-NRF2 interaction in non-small-cell lung cancer. PLoS Med. 2006, 3, e420. [CrossRef] 
8. Meng, H.; Guo, J.; Wang, H.; Yan, P.; Niu, X.; Zhang, J. Erythropoietin activates Keap1-Nrf2/ARE pathway in rat brain after ischemia. Int. J. Neurosci. 2014, 124, 362-368. [CrossRef]

9. Cai, M.C.; Chen, M.; Ma, P.; Wu, J.; Lu, H.; Zhang, S.; Liu, J.; Zhao, X.; Zhuang, G.; $\mathrm{Yu}, \mathrm{Z}$.; et al. Clinicopathological, microenvironmental and genetic determinants of molecular subtypes in KEAP1/NRF2-mutant lung cancer. Int. J. Cancer 2019, 144, 788-801. [CrossRef]

10. Gacesa, R.; Dunlap, W.C.; Barlow, D.J.; Laskowski, R.A.; Long, P.F. Rising levels of atmospheric oxygen and evolution of Nrf2. Sci. Rep. 2016, 6, 27740. [CrossRef]

11. Gacesa, R.; Dunlap, W.C.; Long, P.F. Bioinformatics analyses provide insight into distant homology of the Keap1-Nrf2 pathway. Free Radic. Biol. Med. 2015, 88, 373-380. [CrossRef] [PubMed]

12. Mhamdi, A.; Van Breusegem, F. Reactive oxygen species in plant development. Development 2018, 145, dev164376. [CrossRef] [PubMed]

13. Noctor, G.; Reichheld, J.-P.; Foyer, C.H. ROS-related redox regulation and signaling in plants. Semin. Cell Dev. Biol. 2018, 80, 3-12. [CrossRef] [PubMed]

14. Lu, Y.; Ye, X.; Guo, R.; Huang, J.; Wang, W.; Tang, J.; Tan, L.; Zhu, J.K.; Chu, C.; Qian, Y. Genome-wide targeted mutagenesis in rice using the CRISPR/Cas9 system. Mol. Plant 2017, 10, 1242-1245. [CrossRef]

15. Murashige, T.; Skoog, F. A revised medium for rapid growth and bioassays with tobacco tissue cultures. Physiol. Plant. 1962, 15, 473-497. [CrossRef]

16. Leppek, K.; Das, R.; Barna, M. Functional 5' UTR mRNA structures in eukaryotic translation regulation and how to find them. Nat. Rev. Mol. Cell Biol. 2018, 19, 158-174. [CrossRef]

17. Guo, Z.; Mo, Z. Keap1-Nrf2 signaling pathway in angiogenesis and vascular diseases. J. Tissue Eng. Regen. Med. 2020, 14, 869-883. [CrossRef]

18. Watai, Y.; Kobayashi, A.; Nagase, H.; Mizukami, M.; McEvoy, J.; Singer, J.D.; Itoh, K.; Yamamoto, M. Subcellular localization and cytoplasmic complex status of endogenous Keap1. Genes Cells. 2007, 12, 1163-1178. [CrossRef]

19. Tenhaken, R.; Doerks, T.; Bork, P. DCD—A novel plant specific domain in proteins involved in development and programmed cell death. BMC Bioinf. 2005, 6, 1-6. [CrossRef]

20. Zou, M.; Guan, Y.; Ren, H.; Zhang, F.; Chen, F. A bZIP transcription factor, OsABI5, is involved in rice fertility and stress tolerance. Plant Mol. Biol. 2008, 66, 675-683. [CrossRef]

21. Nakamura, S.; Lynch, T.J.; Finkelstein, R.R. Physical interactions between ABA response loci of Arabidopsis. Plant J. 2001, 26, 627-635. [CrossRef]

22. NCBI. Available online: https://www.ncbi.nlm.nih.gov/ (accessed on 30 July 2016).

23. Gramene. Available online: http://www.gramene.org/ (accessed on 23 August 2016).

24. RAPDB. Available online: https://rapdb.dna.affrc.go.jp/index.html (accessed on 24 August 2016).

25. OrthoDB. Available online: https://www.orthodb.org/ (accessed on 14 August 2016).

26. UNAFold. Available online: http://unafold.rna.albany.edu/ (accessed on 14 August 2016).

27. RiceXPro. Available online: http://ricexpro.dna.affrc.go.jp/GGEP/index.php/ (accessed on 14 August 2016).

28. Skubacz, A.; Daszkowska-Golec, A.; Szarejko, I. The role and regulation of ABI5 (ABA-Insensitive 5) in plant development, abiotic stress responses and phytohormone crosstalk. Front. Plant Sci. 2016, 7, 1884. [CrossRef] [PubMed]

29. Liu, S.; Jiang, J.; Liu, Y.; Meng, J.; Xu, S.; Tan, Y.; Li, Y.; Shu, Q.; Huang, J. Characterization and evaluation of OsLCT1 and OsNramp5 mutants generated through CRISPR/Cas9-mediated mutagenesis for breeding low Cd rice. Rice Sci. 2019, 26, 88-97. [CrossRef] [PubMed]

30. Zhang, H.L.; Huang, J.Z.; Chen, X.Y.; Tan, Y.Y.; Shu, Q.Y. Competitive amplification of differentially melting amplicons facilitates efficient genotyping of photoperiod- and temperature-sensitive genic male sterility in rice. Mol. Breed. 2014, 34, 1765-1776. [CrossRef]

31. Li, S.; Liu, S.; Liu, Y.; Lu, H.; Tan, Y.; Huang, J.; Wei, P.; Shu, Q.Y. HRM-facilitated rapid identification and genotyping of mutations induced by CRISPR/Cas9 mutagenesis in rice. Crop Breed. Appl. Biotechnol. 2018, 18, 184-191. [CrossRef]

32. Basnet, R.; Zhang, J.; Hussain, N.; Shu, Q. Characterization and mutational analysis of a monogalactosyldiacylglycerol synthase gene OsMGD2 in rice. Front. Plant Sci. 2019, 10, 1-12. [CrossRef]

33. He, F.; Chen, S.; Ning, Y.; Wang, G.-L. Rice (Oryza sativa) protoplast isolation and its application for transient expression analysis. Curr. Protoc. Plant Biol. 2016, 1, 373-383. [CrossRef] 
34. Lee, S.-K.; Eom, J.-S.; Hwang, S.-K.; Shin, D.; An, G.; Okita, T.W.; Jeon, J.-S. Plastidic phosphoglucomutase and ADP-glucose pyrophosphorylase mutants impair starch synthesis in rice pollen grains and cause male sterility. J. Exp. Bot. 2016, 67, 5557-5569. [CrossRef]

35. Jiang, M.; Jiang, J.; Li, S.; Li, M.; Tan, Y.; Song, S.; Shu, Q.; Huang, J. Glutamate alleviates cadmium toxicity in rice via suppressing cadmium uptake and translocation. J. Hazard. Mater. 2020, 384, 121319. [CrossRef]

36. He, J.; Duan, Y.; Hua, D.; Fan, G.; Wang, L.; Liu, Y.; Chen, Z.; Han, L.; Qu, L.-J.; Gong, Z. DEXH box RNA helicase-mediated mitochondrial reactive oxygen species production in arabidopsis mediates crosstalk between abscisic acid and auxin signaling. Plant Cell 2012, 24, 1815-1833. [CrossRef]

Publisher's Note: MDPI stays neutral with regard to jurisdictional claims in published maps and institutional affiliations.

(C) 2020 by the authors. Licensee MDPI, Basel, Switzerland. This article is an open access article distributed under the terms and conditions of the Creative Commons Attribution (CC BY) license (http://creativecommons.org/licenses/by/4.0/). 